\title{
Aiding Program Comprehension by Static and Dynamic Feature Analysis
}

\author{
Thomas Eisenbarth, Rainer Koschke, Daniel Simon \\ Universität Stuttgart, Breitwiesenstr. 20-22, 70565 Stuttgart, Germany \\ \{eisenbts, koschke, simondl\}@informatik.uni-stuttgart.de
}

\begin{abstract}
Understanding a system's implementation without prior knowledge is a hard task for reengineers in general. However, some degree of automatic aid is possible. In this paper, we present a technique building a mapping between the system's externally visible behavior and the relevant parts of the source code. Our technique combines dynamic and static analyses to rapidly focus on the system's parts urgently required for a goal-directed process of program understanding.
\end{abstract}

\section{Introduction}

Understanding how a certain feature is implemented is a major problem of program understanding, especially when the understanding is directed to a certain goal like changing or extending the feature. Before real understanding starts, one has to localize the implementation of the feature in the code. Systems often appear as a large number of modules each containing hundreds of lines of code. It is in general not obvious which components implement a given feature. Typically any existing documentation is outdated, the system's original architects are no longer available or their view is outdated due to changes made by others.

One option is to completely reverse engineer the system in order to exhaustively identify its components. We integrated published automatic techniques for component retrieval in an incremental semi-automatic process, in which the results of selected automatic techniques are validated by the user [13].

However, the problem of assigning features to components is not solved by such an exhaustive analysis. Besides, components implementing a specific set of features suffice in many cases, so exhaustive methods are not cost-effective and feature-oriented search focusing on the components of interest is needed.

This paper describes a process and its supporting techniques to identify components implementing a specific set of related features. The process is automated to a great extent. It combines static and dynamic analyses and uses concept analysis - a mathematical technique to investigate binary relations - to derive correspondences between features and components.

\subsection{Terminology}

A feature $f$ is a realized functional requirement (the term feature is intentionally defined weakly because its exact meaning depends on the specific context). Generally, the term feature also subsumes non-functional requirements. However, in the context of this paper only functional features are relevant, i.e., we consider a feature an observable result of value to a user.

A scenario $s$ is a sequence of user inputs triggering actions of a system that yields an observable result to an actor [3]. A scenario is said to execute a feature if the observable result is executed by the scenario's actions. A scenario may execute multiple features. Scenarios resemble use cases but do not include options or choices, so a use case subsumes multiple scenarios.

A component is a computational unit of a system. Components consist of an interface which offers the services of the component and the implementation of these services. The services of the component coherently contribute to the purpose of the component. We address subprograms as well as sets of subprograms as components. The result of our technique are sets of subprograms implementing features.

A subprogram is a function or procedure according to the programming language. Subprograms are the lowestlevel kind of components.

The execution summary of a given program run lists all subprograms called during the run. The execution trace lists the sequence of all performed calls.

A feature-component map describes which components implement a given set of relevant features.

\subsection{Overview}

The remainder of this article is organized as follows. Section 2 starts with related research and gives a brief overview on the process presented in this article, Section 3 briefly introduces concept analysis. Section 4 explains how concept analysis can be used to derive the correspondence of scenarios executing features and components, Section 5 describes how static information can be incorporated. Section 6 shows our implementation of the technique and Section 7 describes our experiences in a case study. Section 8 concludes the paper. 


\section{Related Research on Localizing Features}

Chen and Rajlich [5] propose a semi-automatic method for feature localization, in which an analyst browses the statically derived system dependency graph (SDG) [9]. The SDG describes detailed dependencies among subprograms, types, and variables at the level of individual expressions and statements. Even though navigation on the SDG is computer-aided, the analyst takes on all the search for a feature's implementation. Thus, this method is less suited to quickly and cheaply localize features if it starts without any pre-knowledge on where to begin searching.

Moreover, the method relies on the SDG's quality. If the SDG includes overoptimistic assumptions on function pointers, the analyst may miss functions called via functions pointers. If it reflects too conservative assumptions, the search space increases drastically. It is statically undecidable which control flow paths are taken at runtime, so that every conservative static analysis will yield an overestimated search space. In contrast dynamic analyses exactly tell which parts are really used at runtime - though only for a particular run. However, dynamic analyses recording the execution trace only view the system as a black box giving no insights in internal aspects, like conditions under which subprograms are called.

Wilde and Scully [21] use a dynamic analysis to localize features as follows:

1. The invoking input set I (i.e., a set of test cases or - in our terminology - a set of scenarios) is identified that will invoke a feature.

2. The excluding input set $E$ is identified that will not invoke a feature.

3. The program is executed twice using $I$ and $E$ separately.

4. By comparison of the two resulting execution traces, the subprograms can be identified that implement the feature.

Wilde and Scully focus on localizing specifically needed rather than all required components. For deriving all required components, the execution trace for the including input set is sufficient. By subtracting all subprograms in the execution trace for the excluding input set from those in the execution trace for the invoking input set, only those subprograms remain that specifically deal with the feature. This information alone is not sufficient to identify the interface and the constituents of a component in the source code, but these subprograms are at least a starting point for a more detailed static analysis.

However, Wilde and Scully's approach deals with one feature at a time and gives little insight into connections between a set of related features. If a set of related features is to be considered rather than a single feature, one could repeat the analysis using each feature separately and then unite the specifically required subprograms. However, even then relationships among pairs of features cannot be identified.

Our own contribution. Our technique combines static and dynamic analyses to identify the components implementing a set of related features. Dynamic information by way of execution summaries generated by a profiler for different scenarios is used to identify the subprograms executed when any of the given features is invoked, similarly to Wilde and Scully. One scenario represents the invocation of preferably one single feature only and yields all subprograms executed for this feature.

Beyond simply localizing all required subprograms, we use concept analysis to derive detailed relationships between features and executed subprograms. These relationships identify subprograms jointly required by any subset of features, classify subprograms as low-level or high-level with respect to the given set of features, reveal additional dependencies between subprograms, and help to identify the subprograms that together constitute a larger component during static analysis.

The information gained by concept analysis is then used to guide a subsequent static analysis along the static dependency graph in order to narrow the executed subprograms to those that form self-contained and understandable feature-specific components. Subprograms that are only utility subprograms used as building blocks for a component but not containing any application-specific logic are sorted out. Additional static analyses, like strongly connected component identification, dominance analysis, and program slicing [9] support the search for the components of interest.

The general process is as follows (explanations follow):

1. identify the set of relevant features $F=\left\{f_{1} . . f_{n}\right\}$

2. identify scenarios $\mathcal{A}=\left\{S_{1} . . S_{q}\right\}$ so that the features in $F$ are covered

3. generate execution summaries (profiler); step 3 yields all required subprograms $O=\left\{s_{1} \ldots s_{p}\right\}$ for each scenario

4. create relation table $\mathcal{R}$ such that $\left(S_{1}, s_{1}\right),\left(S_{1}, s_{2}\right), .$. , $\left(S_{q}, s_{p}\right) \in \mathcal{R}$

5. perform concept analysis for $(O, \mathcal{A}, \mathcal{R})$

6 . identify relationships between scenarios and subprograms

7. perform static dependency analyses

In [6], we explained the first six steps of this process based on dynamic information only that results in a feature-component map. This article extends the process by additionally exploiting static analyses and by providing 
another case study.

Applicability. The retrieval of the feature-component map is based on dynamic information where all subprograms are collected that are executed when a feature is invoked. Invoking externally visible features is comparatively simple when a graphical user interface is available. Then, usually only a menu selection or a similar interaction is necessary. In the case of a batch system, one has to vary command line switches and to provide different sets of test data to invoke a feature. However, one might need some knowledge on internal details of a system in order to find suitable test data.

Our technique is primarily suited for functional features that may be mapped to components. In particular non-functional features do not easily map to components. For example, features would have to take time into account for applications where timing is critical (because it may result in different behavior).

The technique is not suited for features that are only internally visible, like a program using a garbage collector. Strictly speaking, internal features may be viewed as implementation details. It is not clear how to execute internal features from outside and how to derive from an execution summary how these features are implemented - or if they are implemented at all.

\section{Concept Analysis}

Concept analysis is a mathematical technique that provides insights into binary relations. The mathematical foundation of concept analysis was laid by Birkhoff in 1940. Primarily Snelting has recently introduced concept analysis to software engineering. Since then it has been used to evaluate class hierarchies [19], explore configuration structures of preprocessor statements [14, 18], for redocumentation [15], and to recover components $[4,8,10,16,17,20]$.

The binary relation in our specific application of concept analysis to derive the scenario-subprogram relationships states which subprograms are required when a feature is invoked.

Concept analysis is based on a relation $\mathcal{R}$ between a set of objects $O$ and a set of attributes $\mathcal{A}$, hence $\mathcal{R} \subseteq O \times \mathcal{A}$.

The tuple $C=(O, \mathcal{A}, \mathcal{R})$ is called formal context. For a set of objects $O \subseteq O$ the set of common attributes $\sigma$ is defined as:

$$
\sigma(O)=\{a \in \mathcal{A} \mid \forall(o \in O)(o, a) \in R
$$

Analogously, the set of common objects $\tau$ for a set of attributes $A \subseteq \mathcal{A}$ is defined as:

$$
\tau(A)=\{o \in O \mid \forall(a \in A)(o, a) \in R
$$

In Section 4.1 the formal context for applying concept analysis to derive the scenario-subprogram relationships will be laid down as follows;

- subprograms will be considered objects,

- scenarios will be considered attributes,

- a pair (subprogram s, scenario $S$ ) is in relation $R$ if $s$ is executed when $S$ is performed.

However, here - for the time being - we will use the binary relation between arbitrary objects and attributes shown in Table 1 as an abstract example. An object $o_{i}$ has attribute $a_{j}$ if row $i$ and column $j$ is marked with an $\times$ in Table 1 (the example stems from Lindig and Snelting [10]). For this table, also known as relation table, the following equations hold:

$$
\begin{aligned}
& \sigma\left(\left\{o_{1}\right\}\right)=\left\{a_{1}, a_{2}\right\} \\
& \tau\left(\left\{a_{7}, a_{8}\right\}\right)=\left\{o_{3}, o_{4}\right\}
\end{aligned}
$$

\begin{tabular}{|c|c|c|c|c|c|c|c|c|}
\hline & $\mathrm{a}_{1}$ & $\mathrm{a}_{2}$ & $\mathrm{a}_{3}$ & $\mathrm{a}_{4}$ & $\mathrm{a}_{5}$ & $\mathrm{a}_{6}$ & $\mathrm{a}_{7}$ & $\mathrm{a}_{8}$ \\
\hline $\mathrm{o}_{1}$ & $\times$ & $\times$ & & & & & & \\
$\mathrm{o}_{2}$ & & & $\times$ & $\times$ & $\times$ & & & \\
$\mathrm{o}_{3}$ & & & $\times$ & $\times$ & & $\times$ & $\times$ & $\times$ \\
$\mathrm{o}_{4}$ & & & $\times$ & $\times$ & $\times$ & $\times$ & $\times$ & $\times$ \\
\hline
\end{tabular}

Table 1: Example relation.

A pair $(O, A)$ is called concept if $A=\sigma(O) \wedge O=\tau(A)$ holds, i.e., all objects share all attributes. For a concept $c=$ $(O, A), O$ is the extent of $c$, denoted by extent $(c)$, and $A$ is the intent of $c$, denoted by intent $(c)$.

Informally, a concept corresponds to a maximal rectangle of filled table cells modulo row and column permutations. For example, Table 2 contains the concepts for the relation in Table 1.

$$
\begin{array}{|c|c|}
\hline \mathrm{C}_{1} & \left(\left\{\mathrm{o}_{1}, \mathrm{o}_{2}, \mathrm{o}_{3}, \mathrm{o}_{4}\right\}, \varnothing\right) \\
\mathrm{C}_{2} & \left(\left\{\mathrm{o}_{2}, \mathrm{o}_{3}, \mathrm{o}_{4}\right\},\left\{\mathrm{a}_{3}, \mathrm{a}_{4}\right\}\right) \\
\mathrm{C}_{3} & \left(\left\{\mathrm{o}_{1}\right\},\left\{\mathrm{a}_{1}, \mathrm{a}_{2}\right\}\right) \\
\mathrm{C}_{4} & \left(\left\{\mathrm{o}_{2}, \mathrm{o}_{4}\right\},\left\{\mathrm{a}_{3}, \mathrm{a}_{4}, \mathrm{a}_{5}\right\}\right) \\
\mathrm{C}_{5} & \left(\left\{\mathrm{o}_{3}, \mathrm{o}_{4}\right\},\left\{\mathrm{a}_{3}, \mathrm{a}_{4}, \mathrm{a}_{6}, \mathrm{a}_{7}, \mathrm{a}_{8}\right\}\right) \\
\mathrm{C}_{6} & \left(\left\{\mathrm{o}_{4}\right\},\left\{\mathrm{a}_{3}, \mathrm{a}_{4}, \mathrm{a}_{5}, \mathrm{a}_{6}, \mathrm{a}_{7}, \mathrm{a}_{8}\right\}\right) \\
\mathrm{C}_{7} & \left(\varnothing,\left\{\mathrm{a}_{1}, \mathrm{a}_{2}, \mathrm{a}_{3}, \mathrm{a}_{4}, \mathrm{a}_{5}, \mathrm{a}_{6}, \mathrm{a}_{7}, \mathrm{a}_{8}\right\}\right) \\
\hline
\end{array}
$$

Table 2: Concepts for Table 1.

The set of all concepts of a given formal context forms a partial order via:

$$
\begin{aligned}
& \left(O_{1}, A_{1}\right) \leq\left(O_{2}, A_{2}\right) \Leftrightarrow O_{1} \subseteq O_{2} \text { or equivalently with } \\
& \left(O_{1}, A_{1}\right) \leq\left(O_{2}, A_{2}\right) \Leftrightarrow A_{1} \supseteq A_{2} .
\end{aligned}
$$

If $c_{1} \leq c_{2}$ holds, then $c_{1}$ is called a subconcept of $c_{2}$ and $c_{2}$ is called superconcept of $c_{1}$. For instance, $\left(\left\{o_{2}, o_{4}\right\},\left\{a_{3}, a_{4}, a_{5}\right\}\right) \leq\left(\left\{o_{2}, o_{3}, o_{4}\right\},\left\{a_{3}, a_{4}\right\}\right)$ is true in 
Table 2.

The set $\mathcal{L}$ of all concepts of a given formal context and the partial order $\leq$ form a complete lattice, called concept lattice:

$$
\mathcal{L}(C)=\left\{(O, A) \in 2^{O} \times 2^{\mathcal{A}} \mid A=\sigma(O) \wedge O=\tau(A)\right\}
$$

The infimum of two concepts in this lattice is computed by intersecting their extents as follows:

$$
\left(O_{1}, A_{1}\right) \wedge\left(O_{2}, A_{2}\right)=\left(O_{1} \cap O_{2}, \sigma\left(O_{1} \cap O_{2}\right)\right)
$$

The infimum describes a set of common attributes of two sets of objects. Similarly, the supremum is determined by intersecting the intents:

$$
\left(O_{1}, A_{1}\right) \vee\left(O_{2}, A_{2}\right)=\left(\tau\left(A_{1} \cap A_{2}\right), A_{1} \cap A_{2}\right)
$$

The supremum ascertains the set of common objects, which share all attributes in the intersection of two sets of attributes.

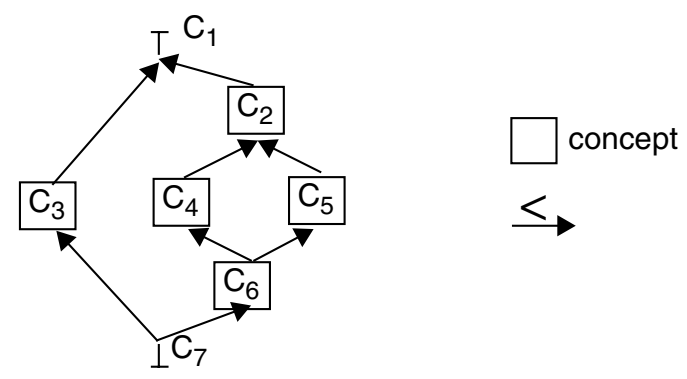

Figure 1. Concept lattice for Table 1.

The concept lattice for the example relation in Table 1 can be graphically depicted as a directed acyclic graph whose nodes represent concepts and whose edges denote the superconcept/subconcept relation $<$ as shown in Figure 1. The most general concept is called the top element and is denoted by $T$. The most special concept is called the bottom element and is denoted by $\perp$.

The combination of the graphical representation in Figure 1 and the contents of the concepts in Table 2 form the concept lattice. The complete information can be visualized in a more readable equivalent way by marking only the graph node with an attribute $a \in \mathcal{A}$ whose represented concept is the most general concept that has $a$ in its intent. Analogously, a node will be marked with an object $o \in O$ if it represents the most special concept that has $o$ in its extent. The unique element $\mu$ in the concept lattice marked with $a$ is therefore:

$$
\mu(a)=\bigvee\{c \in \mathcal{L}(C) \mid a \in \operatorname{intent}(c)\}
$$

The unique element $\gamma$ marked with object $o$ is:

$$
\gamma(o)=\wedge\{c \in \mathcal{L}(C) \mid o \in \operatorname{extent}(c)\}
$$

We will call a graph representing a concept lattice using this marking strategy a sparse representation of the lattice. The equivalent sparse representation for Figure 1 is shown in Figure 2. The content of a node $N$ in this representation can be derived as follows:

- the objects of $N$ are all objects at and below $N$,

- the attributes of $N$ are all attributes at and above $N$.

For instance, the node in Figure 2 marked with $o_{2}$ and $a_{5}$ is the concept $\left(\left\{\mathrm{o}_{2}, \mathrm{o}_{4}\right\},\left\{\mathrm{a}_{3}, \mathrm{a}_{4}, \mathrm{a}_{5}\right\}\right)$.

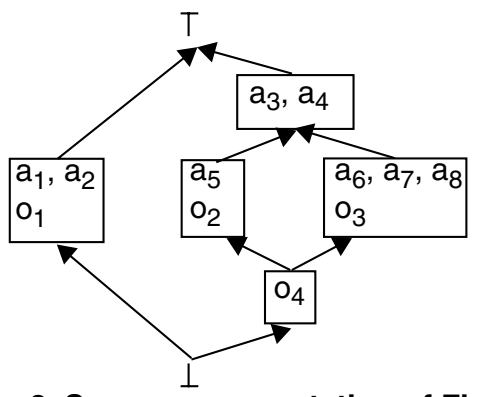

Figure 2. Sparse representation of Figure 1.

\section{Dynamic Analysis}

In order to derive the feature-component map via concept analysis, one has to define the formal context (objects, attributes, relation) and to interpret the resulting concept lattice accordingly.

\subsection{Context for Scenarios and Subprograms}

The goal of the dynamic analysis is to find out which subprograms contribute to a given set of features. For each feature, a scenario is prepared that exploits this feature. Hence, subprograms will be considered objects of the formal context, whereas scenarios will be considered attributes. In the reverse case, the concept lattice is simply inverted but the derived information will be the same.

The relation for the formal context necessary for concept analysis is thus defined as follows:

$(s, S) \in \mathcal{R}$ if and only if subprogram $s$ is required for scenario $S$; a subprogram is required when it needs to be executed.

In order to obtain the relation, a set of scenarios needs to be prepared where each scenario executes preferably only one relevant feature. Then the system is used according to the set of scenarios, one at a time, and the execution summaries are recorded. Each system run yields all required subprograms for a single scenario, i.e., one column of the relation table can be filled per system run. Applying all scenarios provides the complete relation table.

\subsection{On Features and Scenarios}

Because one feature can be invoked by many scenarios and one scenario can invoke several features, there is not 
always a strict correspondence between features and scenarios. If there is an $n: m$ mapping between scenarios and features, one has to locate the concepts in the lattice where scenarios contributing to a feature overlap. Assume we analyze a drawing tool and features are the ability to draw different types of objects, like circles, rectangle, etc., and the ability to apply different actions on drawn objects, like move, rotate, or scale. Let us further assume that we have four scenarios: scenario $S_{A}$ is "draw a circle and move it", $S_{B}$ is "draw a circle and scale it", $S_{C}$ is "draw a rectangle and move it", and $S_{D}$ is "draw a rectangle and scale it". In the concept lattice for these scenarios, the concept including $S_{A}$ and $S_{C}$ will include all subprograms related to the feature move whereas the concept including $S_{B}$ and $S_{D}$ contains the subprograms for the scaling feature. The concept including $S_{A}$ and $S_{B}$ includes all subprograms needed to draw circles, the concept including $S_{C}$ and $S_{D}$ includes all subprograms related to rectangles. Because features are combined in scenarios, one has to interpret the results revealed by the concept lattice. For instance, if the system is implemented in an object-oriented style in which the actions on each object type are implemented by a separate subprogram, one will get concepts each including one object type and one action. Presumably, there are some subprograms needed for all operations on circles (like drawing and hiding), which will go into one subconcept (see Figure 3).

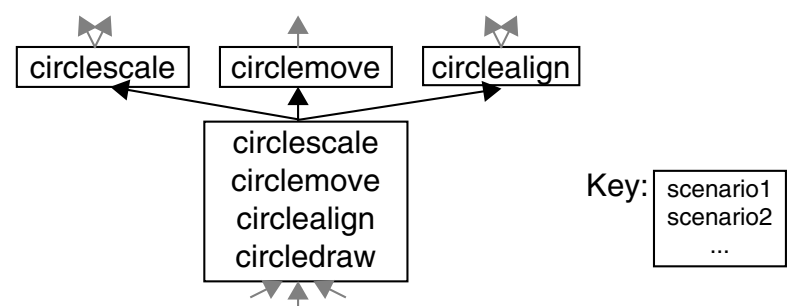

Figure 3. Concept lattice A.

In an alternative functional-style implementation, in which each subprogram implements actions on different types of objects, one will get one concept for each action including scenarios for all object types (see Figure 4). Interestingly enough, the concept lattice will thus show whether an object-oriented or functional-style implementation was chosen.

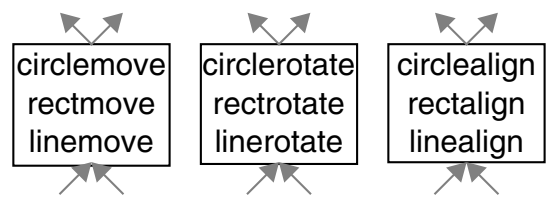

Figure 4. Alternative concept lattice B.

In most cases the relationship between scenarios and features is a 1:1 mapping or is at least intuitively clear from the concept lattice. Consequently, we will assume that a scenario can easily be mapped onto a feature in the following.

\subsection{Interpretation of the Concept Lattice}

Concept analysis applied to the formal context described in the last section yields a lattice, from which interesting relationships can be derived. These relationships can be fully automatically derived and presented to the analyst such that the theoretical background can be hidden. The only thing an analyst has to know is how to interpret the derived relationships. This section explains how interesting relationships can be derived automatically.

As already abstractly described in Section 3 the following base relationships can be derived from the sparse representation of the lattice (note the duality):

- A subprogram $s$ is required for all scenarios at and above $\gamma(s)$ - as defined by EQ(1) on page 4 - in the lattice.

- A scenario $S$ requires all subprograms at and below $\mu(S)$ - as defined by EQ(2) on page 4 - in the lattice.

- A subprogram $s$ is specific to exactly one scenario $S$ if $S$ is the only scenario on all paths from $\gamma(s)$ to the top element.

- A scenario $S$ is specific to exactly one subprogram $s$ if $s$ is the only subprogram on all paths from $\mu(S)$ to the bottom element (i.e, $s$ is the only subprogram required to implement scenario $S$ ).

- Scenarios to which two subprograms $s_{1}$ and $s_{2}$ jointly contribute can be identified by $\gamma\left(s_{1}\right) \vee \gamma\left(s_{2}\right)$. In the lattice, it is the closest common node toward the top element starting at the nodes to which $s_{1}$ and $s_{2}$ are attached. All scenarios at and above this common node are those jointly implemented by $s_{1}$ and $s_{2}$.

- Subprograms jointly required for two scenarios $S_{1}$ and $S_{2}$ are described by $\mu\left(S_{1}\right) \wedge \mu\left(S_{2}\right)$. In the lattice, it is the closest common node toward the bottom element starting at the nodes to which $S_{1}$ and $S_{2}$ are attached. All subprograms at and below this common node are those jointly required for $S_{1}$ and $S_{2}$.

- Subprograms required for all scenarios can be found at the bottom element.

- Scenarios that require all subprograms can be found at the top element.

Beyond these relationships between subprograms and scenarios, further useful aspects between scenarios on one hand and between subprograms on the other hand may be derived: 
- If $\gamma\left(s_{1}\right)<\gamma\left(s_{2}\right)$ holds for two subprograms $s_{1}$ and $s_{2}$, then subprogram $s_{2}$ is more specific with respect to the given use case than subprogram $s_{1}$ because $s_{1}$ contributes not just to the features for which $s_{2}$ contributes, but also to other features.

- If $\mu\left(S_{1}\right)<\mu\left(S_{2}\right)$ holds for two scenarios $S_{1}$ and $S_{2}$, then scenario $S_{2}$ is based on scenario $S_{1}$ because if $S_{2}$ is executed, all subprograms in the extent of $\mu\left(S_{1}\right)$ need also to be executed.

Thus the lattice also reflects the level of application specificity. The information described above can be derived by a tool and fed back to the analyst. Inspecting the relationships derived from the concept lattice, a decision may be made to analyze only a subset of the original features in depth due to the additional dependencies that concept analysis could reveal. All subprograms required for these features (easily derived from the concept lattice) form a starting point for further static analyses to identify components, to investigate quality (like maintainability, extractability, and integrability) and to estimate effort for subsequent steps.

\section{Static Dependency Analysis}

From the concept lattice, we can easily derive all subprograms executed for any set of relevant features (note that we use features and scenarios as synonyms from here on, see Section 4.2). However, this gives us only a set of subprograms, but it is not clear which of these subprograms form a feature-specific component and which of them are general-purpose subprograms that are only used as building blocks for other components but do not contain any feature-specific logic. Given a feature $f$ of interest this question can be answered as follows:

- As a first approximation, all subprograms in the extent of concept $\mu(f)$ according to $\mathrm{EQ}(2)$ on page 4 may jointly constitute a component.

- Irrelevant subprograms among these subprograms can be sorted out by a goal-directed manual inspection.

\subsection{Building the Starting Set}

All subprograms in the extent of a concept jointly contribute to all features in the intent of the concept, which immediately follows from the definition of a concept. However, there may also be subprograms in the extent that contribute to other features as well, so they are not specific to the given feature. There may be subprograms in the extent that do not contain any feature-specific code at all. Thus, subprograms in the extent of the concept need to be inspected manually. Because there are no reliable criteria known that distinguish feature-specific code from generalpurpose code, this analysis cannot be automated and human expertise is necessary. However, the concept lattice may narrow the candidates for manual inspection.

The concept lattice and the dependency graph can help to decide in which order the subprograms are to be inspected such that the effort for manual inspection can be reduced to a minimum. Since we are interested in subprograms most specific to a feature $f$ we start at those subprograms $s$ that are attached to $\mu(f)$, i.e., for which $\mu(f)=\gamma(s)$ holds. If there are no such subprograms, we collect all concepts below $\mu(f)$ with minimal distance from $\mu(f)$ to which subprograms are attached. There can be more than one concept, so we unite all subprograms that are attached to one of these concepts. The subset of subprograms identified in this step and accepted by manual inspection is called the starting set $S(f)$.

\subsection{Inspection of the Static Call Graph}

Next, we inspect all subprograms called from subprograms in $S(f)$. We generate the call graph (as one specific subset of the dependency graph) that contains all subprograms transitively called by subprograms in $S(f)$ as derived by a static analysis. We concentrate on subprograms here because they are the active constituents of a component. Global variables and types may be added once all subprograms have been identified. Subprograms in $S(f)$ are said to be the roots of this call graph. A static points-to analysis is needed to resolve calls via function pointers if present. The static points-to analysis may take advantage of the knowledge about actually called functions yielded by the dynamic analysis.

It is sufficient to consider only those subprograms $s$ for which $s \in$ extent $(\mu(f))$ holds because only those subprograms are actually called when $f$ is invoked according to the dynamic analysis. Hence, we combine static and dynamic information to eliminate conditional static subprogram calls in order to reduce the search space.

If the component for the feature $f$ is to be understood, calls to subprograms not in $\operatorname{extent}(\mu(f))$ can be safely ignored in the original source code in order to cut apparent static dependencies - unless there is another relevant feature relying on the same subprogram and in whose context the call is actually executed. In this case, one can apply slicing techniques to separate the code relevant for each feature.

Once the call graph is generated, it can be traversed to inspect subprograms. Any kind of traversal is possible, but a depth-first search is most suited because a subprogram can only be understood if all its called subprograms are understood. Moreover, in a breadth-first search, a human has to cope with continuous context switches. The goal of 
the inspection is to sort out subprograms that do not belong to the component in a narrow sense because they do not contain feature-specific code. Two additional analyses gather further information useful while navigating on the call graph:

- Strongly connected component analysis is used to identify cycles in the call graph: If there is one subprogram in a cycle that contains feature-specific code, all subprograms of the cycle need to be added to the component because of the cyclic dependency.

- Dominance analysis is used to identify subprograms that are local to other subprograms. A subprogram $s_{1}$ is said to dominate another subprogram $s_{2}$ if every path in the call graph from one of its roots in $S(f)$ to $s_{2}$ contains $s_{1}$. In other words, $s_{2}$ can only be called by way of $s_{1}$. If a subprogram $s$ is found to be feature-specific, then all its dominators also need to be added to the component, because they need to be called in order for $s$ to be executed. If neither of a dominator's dominatees contain feature-specific code and the dominator itself is not feature-specific, then the dominator is a clear cutting point as all its dominatees are local to it. Consequently, the dominator and all its dominatees can be safely omitted while understanding the system.

Inspection is done along the call relation in the call graph rather than following a top-down traversal in the concept lattice because the lattice does not really reflect the dependencies: $\gamma\left(s_{1}\right)>\gamma\left(s_{2}\right)$ does not imply that $s_{1}$ calls $s_{2}$. However, the concept lattice may still provide useful information for the inspection. In Section 4.3 we made the observation that the lower a concept $\gamma(s)$ is in the lattice, the more general subprogram $s$ is as it serves more features - and vice versa. Thus, the concept lattice gives us insight into the level of abstraction of a subprogram and, therefore, contributes to the degree of confidence that a specific subprogram contains feature-specific code.

If more than one feature is relevant, one simply unites the starting sets for each feature and then follows the same approach. For more than one feature, the concept lattice provides insight into feature interaction and identifies subprograms jointly used by several features. Such subprograms can then be considered a component of their own. Hence, not only one component is detected but the call graph is partitioned into several connected components by merging connected concepts in the lattice and by filtering out subprograms in their extent.

Once all subprograms have been identified, static dependency analysis, e.g., program slicing, can be used to extract the components' code including necessary variable and type declarations. Moreover, static dependency analysis will also be used to identify the provided interface of the extracted components - those elements of a component used in other parts of the system - and the required interface - those elements of the system the component's elements rely on and that are not declared by the component itself.

\section{Implementation}

The implementation of detecting the executed subprograms per scenario and applying concept analysis is surprisingly simple (if one already has a tool for concept analysis). Our prototype for a Unix environment is an opportunistic integration of the following parts:

- Gnu C compiler gcc to compile the system using a command line switch for generating profiling information,

- Gnu object code viewer $\mathrm{nm}$ and a short Perl script in order to identify all functions of the system (as opposed to those included from standard libraries),

- Gnu profiler gprof and a short Perl script to ascertain the executed functions in the execution summary,

- concept analysis tool concepts [11],

- graph editor Graphlet [2] to visualize the concept lattice,

- one short Perl script to convert the file formats of concepts and Graphlet (147 LOC),

- and our extended version of Rigi [22].

For deriving the static dependency graph and to identify components, we have developed the Bauhaus toolkit [1]. It allows deriving detailed dependencies as system dependency graph (SDG) [9] and more coarse-grained dependencies as resource flow graph (RFG) [12]. An SDG describes set-use data dependencies and control dependencies at the level of expressions and statements, while an RFG contains only global declarations (global variables, user-defined types, and subprograms) and their relationships (variable access, signature relations, calls, etc.). The RFG is derived from the SDG by abstracting from individual expressions and statements and is better suited for presentation to a human analyst. The Bauhaus toolkit uses Rigi [22] to visualize the RFG. Rigi supports graph navigation and provides immediate access to the original source code for a more detailed investigation. We added multiple additional automatic analyses specifically to support component retrieval [13], like identification of cyclic dependencies and local (i.e., dominated) parts (see Section 5.2).

\section{Case Study}

We analyzed two web browsers (see Table 3 ) using the 
same set of relevant related features. The concept lattice for each of these systems was derived as described in Section 6 . The required subprograms as identified by dynamic analysis and the relationships derived by concept analysis formed a starting point for the static dependency analysis. The static dependency analysis was done on the resource flow graph [12] using the Bauhaus toolkit. The experiences are reported in this section.

\begin{tabular}{|r|r|r|r|}
\hline \multicolumn{1}{|c|}{ System } & Version & KLOC (wc) & \#subprograms \\
\hline Mosaic & 2.6 & 51,440 & 701 \\
Chimera & $2.0 \mathrm{a} 19$ & 38,208 & 928 \\
\hline
\end{tabular}

Table 3: Analyzed web browsers.

\subsection{Case Study Setup}

In two experiments, History and Bookmark, we tried to understand how two specific sets of related features are implemented in both browsers using the process described above. The goal of this analysis was to recover the featurespecific components and the way they interact, i.e., to reverse engineer a partial description of the software architecture. The partial software architecture, for instance, allows one to decide whether feature-specific components can be extracted from one system and integrated into another system with only minor changes. Chimera does not implement all features that Mosaic provides and we wanted to find out whether the respective feature-specific components of Mosaic can be reused for Chimera.

Use case History $(\mathbf{H})$ : Chimera allows going back in the history of already visited URLs. However, Chimera does not have a forward button that allows a user to move forward in the history again after the back button was used. Mosaic has both a back and forward button. In this experiment, going back and going forward were considered related features.

Use case Bookmark (B): Both Mosaic and Chimera offer bookmarks for visited URLs. URLs may be bookmarked, and bookmarked URLs may be loaded and removed. We considered the following related features: addition of a new bookmark for a currently viewed URL, removal of a bookmark, and navigation to a bookmarked URL.

The question we want to answer in our case study was as follows:

Identification and extraction: How are the history and the bookmark features implemented in Mosaic? What are the interfaces between the specific components that implement these features and the rest of Mosaic? Analogously for Chimera's partial implementation of these features. In both cases, a partial description of the software architecture was recovered.

\subsection{Scenarios for Dynamic Analysis}

For each experiment and each browser, we ran the browser in a start-end scenario in which the browser was started and immediately quit in order to separate start-up and shutdown code. The following additional scenarios were defined specifically to the two experiments. Use case History was covered by the following three scenarios:

(H1) basic scenario doing nothing but browsing,

(H2) scenario using the back button, and

(H3) scenario using the back and forward buttons.

For Chimera, the last scenario was not performed (because Chimera possesses no forward button). Use case Bookmark was covered by the following four scenarios:

(B1) basic scenario: simply opening and closing the bookmark window,

(B2) scenario: adding a new bookmark for the currently displayed URL,

(B3) scenario: removing a bookmark,

(B4) scenario: selecting a bookmark and visiting the associated URL.

Each scenario was immediately ended by quitting the respective system. We provided scenarios that use one feature only except for one scenario: One cannot use the forward button without using the back button. Consequently, the concept containing subprograms executed for scenario $(\mathrm{H} 2)$ is a subconcept of the concept related to (H3). Likewise, a bookmark can only be deleted when an URL has been added before. However, to circumvent this problem, we started the browser with a non-empty bookmark file in all scenarios. Thus, we did not consider the case of insertion into an empty bookmark list.

\subsection{Static Dependency Analysis}

In the dependency graph for the browsers (given as RFG, see Section 6), visualized using the Bauhaus extension to Rigi, we derived all statically transitively called functions (using Rigi's basic selection facilities) and intersected the static information with the actually executed functions manually. We additionally filtered out all functions specific to HTML and the X-window-based graphical user interface guided by the browser's proper naming conventions. These functions were all in the bottom element of the concept lattice.

\subsection{Results}

Table 4 provides a summary of the numbers of subpro- 
grams that needed to be further considered in each step and shows how the search space could be reduced in each step. $(\mathrm{H})$ denotes the history, (B) the bookmark experiment. The total number of all functions of the kernels (not including libraries such as html, jpeg, zlib) are in column (1), the number of actually executed subprograms for each scenario is shown in column (2). All functions statically called by subprograms selected from the set of dynamically executed functions in upper concepts of the lattice are in column (3). The intersection of column (2) and (3) is contained in column (2) $\cap(3)$. Column relevant reports all functions in column (2) $\cap(3)$ that are specific to the selected features according to our manual inspection. All other functions are used for other purposes than bookmarks and histories.

\begin{tabular}{|c|c|r|r|r|r|}
\hline & (1) & (2) & \multicolumn{1}{|c|}{ (3) } & (2) $\cap(3)$ & relevant \\
\hline Mosaic/(B) & 701 & 359 & 99 & 74 & 16 \\
Mosaic/(H) & & 348 & 74 & 65 & 6 \\
\hline Chimera/(B) & 928 & 431 & 89 & 55 & 3 \\
Chimera/(H) & & 419 & 123 & 55 & 24 \\
\hline
\end{tabular}

Table 4: Subprogram counts for Mosaic and Chimera

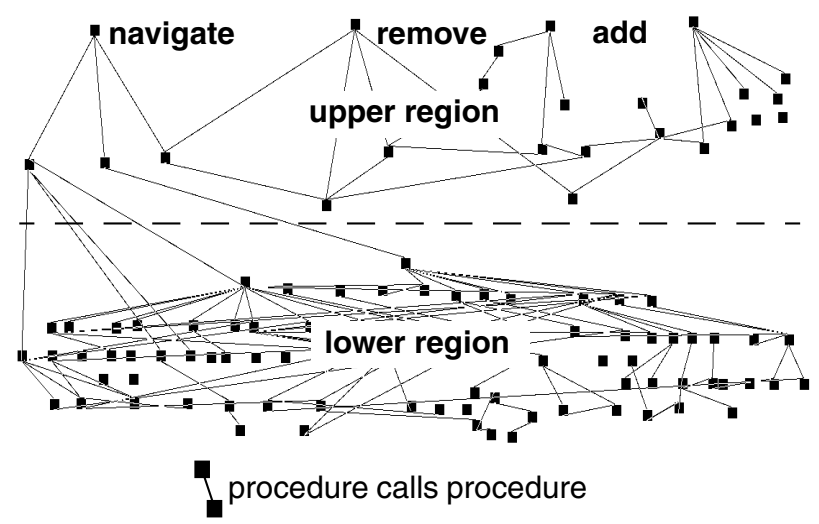

Figure 5. Relevant parts of Chimera for history

Eventually, only a small number of subprograms needed to be inspected more thoroughly due to the topdown inspection process. As an example, Figure 5 shows the remaining subprograms of Chimera (omitting their names) relevant to the history experiment. This picture clearly shows the possible cutting points in the dependency graph between functions specific to the history features (upper region) and non-specific functions (lower region).

We recovered the parts of the architecture of Mosaic and Chimera relevant to the two use cases. The recovered partial architecture shows that Chimera's browser kernel is built around a list of visited URLs whereas Mosaic's browser kernel does not know the history of visited URLs at all.
Results for History. The interface between Mosaic's browser kernel and the history component (see Figure 6) is formed by three subprograms to (a) get the current URL, (b) set the current URL, and (c) communicate the action and event (changed URL).

The history component can be easily extracted from Mosaic's source code because it is a separate component while the history is an integral part of Chimera's kernel. There is no set of subprograms of Chimera that could be reasonably addressed as "history manager component" as in Mosaic. Chimera uses a layer of wrappers calling a dispatching routine around a list of actions where the displayed URLs are part of that list.

As the analysis of the partial architectural architectures reveals, re-using Mosaic's history components in Chimera would be very difficult due to the architectural mismatch [7].

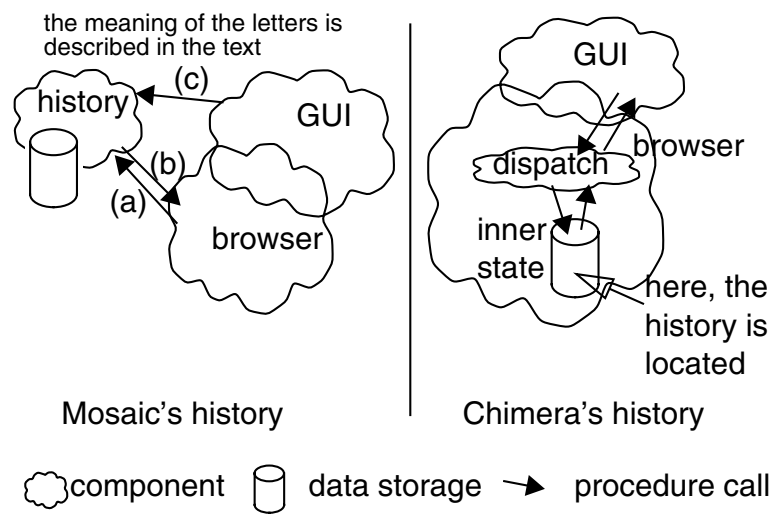

Figure 6. Mosaic's and Chimera's history architecture

Results for Bookmarks. The partial architectures of the two systems are similar to each other with respect to bookmarks. Both architectures include an encapsulated bookmark component, which communicates via a narrow interface with the basic browser kernel (see Figure 7).

The basic actions that have to be performed are: (a) get currently shown URL, (b) set currently shown URL, (c) display the bookmarks, and (d) communicate the bookmark selection back.

Exchanging the two implementations between Mosaic and Chimera would be reasonably easy.

\section{Conclusions}

The technique presented in this paper identifies all components specific to a set of related features using execution traces for different usage scenarios. At first, concept analysis - a mathematically sound technique to analyze binary relations - allows to locate the most feature-specific subprograms among all executed subprograms. Then, a static analysis uses these feature-specific subprograms to 


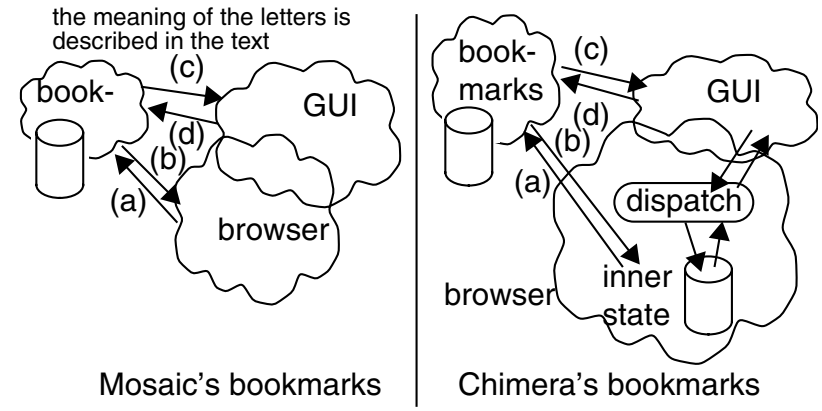

\section{Figure 7. Mosaic's and Chimera's bookmark architec- ture}

identify additional feature-specific subprograms along the dependency graph. The combination of dynamic and static information reduces the search space drastically.

In a case study, analyzing two web browsers, we could recover a partial description of the software architecture with respect to a specific set of related features. Commonalities and variabilities between these partial architectures could be recovered quickly. Altogether, we found 16 and 6 , respectively, feature-specific subprograms out of 701 subprograms for Mosaic and 3 and 24, respectively, out of 928 for Chimera. Only very few subprograms needed to be inspected manually.

Deriving partial architectures with the described technique can support a more goal-oriented and cost-effective program understanding and reverse engineering, thereby facilitating feature-specific re-use and reengineering.

The approach is only applicable to externally visible and executable features, primarily suited for functional features.

\section{References}

[1] Bauhaus project, University of Stuttgart, http://www.informatik.uni-stuttgart.de/ifi/ps/bauhaus.

[2] Brandenburg, F.J., 'Graphlet', Universität Passau, http://www.infosun.fmi.uni-passau.de/Graphlet.

[3] Booch, G., Rumbaugh, Jacobson, J., 'The Unified Modeling Language Reference Manual', Addison-Wesley.

[4] Canfora, G., Cimitile, A., De Lucia, A., Di Lucca, G.A., 'A Case Study of Applying an Eclectic Approach to Identify Objects in Code', Workshop on Program Comprehension, pp. 136-143, 1999.

[5] Chen, K., Rajlich, V., 'Case Study of Feature Location Using Dependence Graph', Proc. of the 8th Int. Workshop on Program Comprehension, pp. 241-249, June 2000.

[6] Eisenbarth, E., Koschke, R. Simon, D., 'Feature-Driven Program Understanding Using Concept Analysis of Execution Traces', Proc. Int. Workshop on Program Comprehension, 2001, to appear.

[7] Garlan, D., Allen, R., Ockerbloom, J., 'Architectural Mismatch or, Why it's hard to build systems out of existing parts", Proceedings of the 17th International Conference on Software Engineering, pp. 179-185, April 1995

[8] Graudejus, H., 'Implementing a Concept Analysis Tool for Identifying Abstract Data Types in C Code', master thesis, University of Kaiserslautern, Germany, 1998.

[9] Horwitz, S., Reps, T., Binkley, D., 'Interprocedural slicing using dependence graphs', ACM Transactions on Programming Languages and Systems, vol. 12, no. 1, pp. 26-60, January 1990.

[10]Lindig, C., Snelting, G., 'Assessing Modular Structure of Legacy Code Based on Mathematical Concept Analysis', Proceedings of the International Conference on Software Engineering, pp. 349-359, 1997.

[11]Lindig, C., Concepts, ftp://ftp.ips.cs.tu-bs.de/pub/local/softech/misc.

[12]Koschke, R., Girard, J.-F., Würthner, M., 'An Intermediate Representation for Reverse Engineering Analyses', Proceedings of the Working Conference on Reverse Engineering, 1998.

[13]Koschke, R., 'Atomic Architectural Component Recovery for Program Understanding and Evolution', Dissertation, Institut für Informatik, Universität Stuttgart, 2000, http://www.informatik.uni-stuttgart.de/ifi/ps/rainer/thesis.

[14]Krone, M., Snelting, G., 'On the Inference of Configuration Structures From Source Code', Proceedings of the International Conference on Software Engineering, pp. 49-57, May 1994.

[15]Kuipers, T., Moonen, L., 'Types and Concept Analysis for Legacy Systems', Proc. Int. Workshop on Program Comprehension, 2001.

[16]Sahraoui, H., Melo. W, Lounis, H., Dumont, F., 'Applying Concept Formation Methods to Object Identification in Procedural Code', Proceedings of the Conference on Automated Software Engineering, pp. 210-218, November 1997.

[17]Siff, M., Reps, T., 'Identifying Modules via Concept Analysis', Proceedings of the International Conference on Software Maintenance, pp. 170-179, October 1997.

[18]Snelting, G., 'Reengineering of Configurations Based on Mathematical Concept Analysis', ACM Transactions on Software Engineering and Methodology vol. 5, no. 2, pp. 146189, April 1997.

[19]Snelting, G., Tip, F., 'Reengineering Class Hierarchies Using Concept Analysis', Proceedings of the ACM SIGSOFT Symposium on the Foundations of Software Engineering, pp. 99110, November 1994.

[20]van Deursen, A., Kuipers, T., 'Identifying objects using cluster and concept analysis', Proc. Int. Conf. Software Engineering, 1999.

[21]Wilde, N., Scully, M.C., 'Software Reconnaissance: Mapping Program Features to Code', Software Maintenance: Research and Practice, vol. 7, pp. 49-62, 1995.

[22]Wong, K., 'The Rigi User's Manual', Version 5.4.4., June 1998. 